\title{
Pregnancy after Aortic Root Replacement in Marfan's Syndrome: A Case Series and Review of the Literature
}

\author{
Dominique Williams, $\mathrm{MD}^{1}$ Kathryn J. Lindley, MD ${ }^{1}$ \\ Harry C. Dietz, MD $^{3} \quad$ Alan C. Braverman, MD $^{1}$ \\ ${ }^{1}$ Cardiovascular Division, Department of Medicine, Washington \\ University School of Medicine, St. Louis, Missouri \\ 2 Department of Obstetrics and Gynecology, Warren Alpert Medical \\ School at Brown University, Providence, Rhode Island \\ ${ }^{3}$ Johns Hopkins University School of Medicine, Howard Hughes \\ Medical Institute, Baltimore, Maryland
}

Am J Perinatol Rep 2018;8:e234-e240.
Melissa Russo, $\mathrm{MD}^{2}$ Jennifer Habashi, $\mathrm{MD}^{3}$

\begin{abstract}
Address for correspondence Alan C. Braverman, MD, Cardiovascular Division, Department of Medicine, Washington University School of Medicine, 660 South Euclid, Campus Box 8086, St. Louis, MO 63110 (e-mail: abraverm@wustl.edu).
\end{abstract}

\begin{abstract}
Keywords

- Marfan's syndrome

- pregnancy

- aortic root replacement

- aortic dissection

Objectives We sought to characterize pregnancy-related aortic complications in women with Marfan's syndrome who had prior aortic root replacement.

Study Design This is a retrospective case series study and literature review of women with Marfan's syndrome with pregnancy after aortic root replacement. We surveyed women with Marfan's syndrome who had successful pregnancy after aortic root replacement using the Marfan Foundation Website and from two large tertiary care Marfan's clinics. Clinical data, counseling information, and details of pregnancy-related aortic complications were compiled. A literature review was performed assessing aortic outcomes in women with Marfan's syndrome with pregnancy after aortic surgery.

Results Fourteen women with 20 pregnancies were identified. Two women had three pregnancies following root replacement for aortic dissection. There were no aortic dissections during the 20 pregnancies. In contrast, aortic dissection was frequently reported in the literature.

Conclusions Women with Marfan's syndrome who become pregnant following aortic root replacement remain at risk for distal aortic dissection related to pregnancy. The exact risk is difficult to quantify but is not zero and women should be counseled accordingly.
\end{abstract}

With medical therapy and preventive aortic surgery, life expectancy in Marfan's syndrome approaches that of the general population. ${ }^{1}$ Unique issues have arisen in caring for this population including counseling women with Marfan's syndrome on the risk of cardiovascular morbidity related to pregnancy. Current literature focuses on the cardiovascular risks of pregnancy prior to aortic surgery. Women with Marfan's syndrome and aortic diameter $<40 \mathrm{~mm}$ are considered to have a relatively low risk $(\leq 1 \%)$ of acute aortic dissection during preg- nancy. ${ }^{2-5}$ However, aortic dissection risk is estimated as high as $10 \%$ in women with aortic root dilation $>40 \mathrm{~mm}$, rapid dilatation, or previous dissection. ${ }^{2,4}$ Women with Marfan's syndrome and aortic dilatation (especially $>45 \mathrm{~mm}$ ) may be counseled to undergo aortic root repair prior to pregnancy to reduce the risk of aortic dissection, 5,6 although this has not been proven to be entirely protective. ${ }^{4,7-10}$

Little is known about the risk of acute aortic dissection related to pregnancy in women with Marfan's syndrome received

June 12, 2018

accepted after revision

August 30, 2018
DOI https://doi.org/

$10.1055 / \mathrm{s}-0038-1675347$. ISSN 2157-6998.
Copyright $\odot 2018$ by Thieme Medical

Publishers, Inc., 333 Seventh Avenue, New York, NY 10001, USA. Tel: +1(212) 584-4662.
License terms

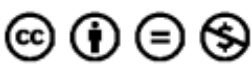


following aortic root replacement. Case reports have documented variable outcomes including successful and uncomplicated pregnancies ${ }^{11-17}$ as well as acute aortic dissection. ${ }^{4,7-10}$ Specific risk factors, coupled with hemodynamic changes and hormonal alternations, which occur during pregnancy and early postpartum may culminate in an acute aortic dissection in susceptible women. Given the paucity of data, it remains difficult to confidently counsel women with Marfan's syndrome with prior aortic root replacement on the risk of acute aortic dissection with pregnancy.

To further study this question, we queried a population of women with Marfan's syndrome with prior aortic root replacement on specific details of each pregnancy following their aortic surgery. We hypothesized that the risk of an aortic dissection related to pregnancy among women with Marfan's syndrome and prior aortic root replacement is low but not zero. We sought to examine potential risk factors for aortic complications related to pregnancy, such as prior aortic dissection, hypertension, lack of $\beta$-blocker use, number of pregnancies, and breastfeeding. We also sought to understand whether there was a significant variation in counseling provided to these women regarding their risk of pregnancy following aortic root replacement.

\section{Methods}

A 32-question survey (see - Supplementary File 1; available only in the online version) regarding the diagnosis of Marfan's syndrome, type of aortic root replacement, history of prior aortic dissection, cardiac and obstetric outcomes of pregnancies, details regarding aortic dissections occurring during pregnancy, and the postpartum period (including timing of dissection [1st, 2nd or 3rd trimester, during labor and delivery, up to 2 weeks postpartum, 2-4 weeks postpartum, or greater than 4 weeks but less than 12 months postpartum]), mode of delivery, method of anesthesia, presence of breastfeeding, use of $\beta$-blockers, presence of hypertension, eclampsia or preeclampsia, smoking, and use of anticoagulants was developed by the investigators and distributed through the Marfan Foundation Website research link and social media Website, www.marfan.org. The survey was tailored to ask the same set of questions for each pregnancy by using branching logic. The survey was distributed on the Marfan Foundation Website and social media site from October 2014 through March 2015. Women with Marfan's syndrome were excluded if they were under the age of 18 years or did not have a pregnancy following aortic root replacement. Washington University in St. Louis School of Medicine (WU) and the Johns Hopkins Hospital (JHH) Marfan's Syndrome Clinics identified additional women with Marfan's syndrome who had pregnancy following aortic root replacement. Pregnancies that resulted in miscarriage or therapeutic abortion were excluded from the final analysis. The institutional review boards at $\mathrm{WU}$, the $\mathrm{JHH}$, and the Marfan Foundation approved the survey.

A literature review was also conducted to identify additional cases of women with Marfan's syndrome with preg- nancy after aortic root replacement. We performed an electronic search using PubMed with the following key terms: aortic root replacement, pregnancy, and Marfan's syndrome. Papers were excluded if it did not include women with Marfan's syndrome with pregnancy after aortic root replacement.

The institutional review boards at WU (IRB 201407102, approved August 2014), the JHH (NA_00037120, approved September 2010, updated November 2017), and the Marfan Foundation approved the survey.

\section{Results}

We identified a total of 14 women with Marfan's syndrome who had 26 pregnancies after aortic root replacement consisting of 20 live births and 6 miscarriages. Seven women with 13 pregnancies were identified through the Marfan Foundation Website. Four women with eight pregnancies were identified from the WU Marfan's Syndrome Clinic. Three women with five pregnancies were identified at the JHH Marfan's Syndrome Clinic (-Table 1 and -Table 2).

Twelve women (85.7\%) had prior elective aortic root replacement for aneurysm disease. Two women (14.3\%) underwent aortic root replacement due to a type A aortic dissection. The mean age at the time of aortic root replacement was $27.4 \pm 4.1$ years. Three $(21.4 \%)$ women reported a history of chronic hypertension prior to pregnancy. One woman without preexisting hypertension developed preeclampsia during pregnancy. $\beta$ blockers were prescribed in 18 of the 20 live birth pregnancies (90\%). Smoking occurred during 3 (15\%) of 20 live birth pregnancies. Antithrombotic (i. e., aspirin, warfarin, enoxaparin) therapy was used in six live birth pregnancies (30\%). Indication for use of antithrombotic therapy was only known in three pregnancies (patients 9 and 14 who had a deep venous thrombosis and mechanical aortic valve replacement, respectively; - Table $\mathbf{1}$ ).

Of the 20 live birth pregnancies, 9 deliveries were by cesarean section (45\%), 8 (40\%) were spontaneous vaginal deliveries, and 3 (15\%) were forcep-assisted vaginal deliveries. Five pregnancies (25\%) were preterm. Eighteen deliveries (90\%) had epidural anesthesia. Breastfeeding for at least 2 weeks occurred in 14 (70\%) live birth pregnancies. Two women (patients 10 and 11) breastfed their infants longer than 12 months.

There were no aortic dissections related to pregnancy or in the immediate weeks postpartum among the women in our series. One woman suffered a fatal type B (descending) aortic dissection 1 year after delivery which was not deemed related to pregnancy because it occurred outside prespecified postpartum period of 1 month (patient 11; - Table 1). Another woman developed a descending thoracic aortic aneurysm requiring open surgical repair 3 years after her second pregnancy (patient 9, - Table 1). Following descending aortic repair, this woman went on to have a successful and uncomplicated third pregnancy and delivery.

All women except one in our series reported receiving some counseling on the risk of aortic dissection with pregnancy following aortic root replacement. Most women were not quoted a specific numerical risk of aortic dissection. Two 


\begin{tabular}{|c|c|c|c|c|c|c|c|c|c|c|c|c|c|c|c|c|c|c|c|c|c|c|c|c|c|c|c|}
\hline 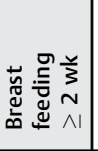 & + & $\Sigma$ & + & I & $\frac{\Delta}{z}$ & $\sum \Sigma$ & $\begin{array}{l}n \\
2\end{array}$ & + & + & $\underline{z}$ & + & + & I & $\underline{z}$ & 1 & 1 & \begin{tabular}{|l|}
0 \\
$E$ \\
0 \\
+ \\
+
\end{tabular} & $\begin{array}{l}\stackrel{\circ}{E} \\
m \\
+ \\
+\end{array}$ & $\begin{array}{l}\stackrel{\circ}{E} \\
m \\
+ \\
+\end{array}$ & $\begin{array}{l}\stackrel{\circ}{E} \\
\stackrel{2}{\sim} \\
\wedge \\
+\end{array}$ & $\begin{array}{l}\stackrel{O}{E} \\
\underset{\sim}{\simeq} \\
\check{+}\end{array}$ & + & $\frac{\pi}{z}$ & + & 1 & & 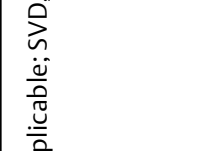 \\
\hline है & 1 & 1 & 1 & I & I & $\begin{array}{lll}1 & 1 \\
\end{array}$ & 11 & 1 & 1 & I & 1 & $\Sigma$ & $\Sigma$ & 1 & 1 & 1 & + & + & + & 1 & 1 & 1 & 1 & 1 & 1 & & $\begin{array}{l}\frac{0}{\pi} \\
\stackrel{0}{0} \\
\stackrel{5}{0} \\
0 \\
0 \\
0\end{array}$ \\
\hline 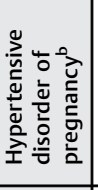 & I & 1 & I & 1 & 1 & \begin{tabular}{l|l}
1 & 1 \\
\end{tabular} & 1 & 1 & 1 & I & 1 & 1 & 1 & 1 & 1 & 1 & 1 & 1 & 1 & 1 & 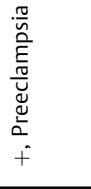 & 1 & 1 & 1 & 1 & & 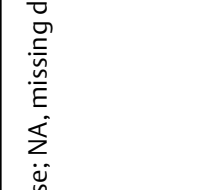 \\
\hline 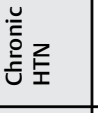 & + & + & + & + & 1 & 11 & I & 1 & 1 & 1 & 1 & 1 & 1 & 1 & 1 & 1 & + & + & + & 1 & 1 & 1 & 1 & 1 & 1 & 1 & $\begin{array}{l}\frac{\pi}{0} \\
\frac{0}{0} \\
\frac{2}{\pi}\end{array}$ \\
\hline 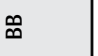 & + & + & + & + & + & ++ & $+\mid+$ & 1 & I & + & + & + & + & + & + & + & + & + & + & + & + & + & + & + & + & + & \\
\hline 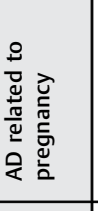 & $\stackrel{\circ}{z}$ & z & 20 & z & 2 & $2 \stackrel{0}{2}$ & $2 \frac{0}{2}$ & $\stackrel{8}{2}$ & $\stackrel{0}{2}$ & $z^{2}$ & 2 & z & i & $\stackrel{0}{2}$ & 2 & 2 & 2 & 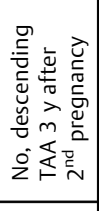 & 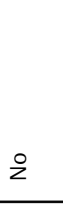 & 2 & 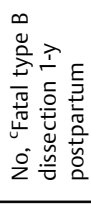 & $i_{1}$ & $\stackrel{0}{z}$ & 2 & $\stackrel{0}{z}$ & $\stackrel{0}{2}$ & 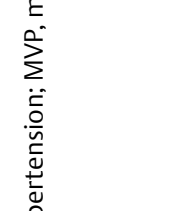 \\
\hline 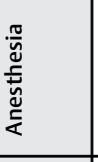 & 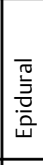 & $\begin{array}{l}\check{1} \\
2 \\
\end{array}$ & 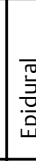 & 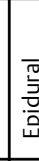 & 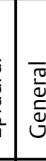 & 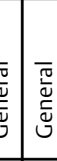 & 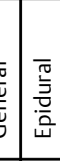 & 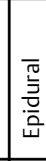 & 焉 & 产 & 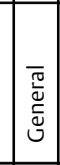 & 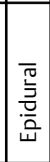 & 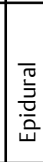 & 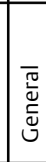 & 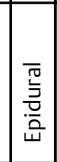 & 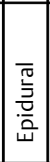 & \begin{tabular}{|l|}
$\overline{0}$ \\
$\frac{0}{3}$ \\
$\overline{2}$ \\
\end{tabular} & $\begin{array}{l}\overline{\frac{\pi}{5}} \\
\overline{\bar{n}} \\
\bar{\omega}\end{array}$ & 嵒 & 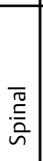 & $\begin{array}{l}\overline{\overline{0}} \\
\overline{\frac{5}{5}} \\
\overline{\mathrm{n}}\end{array}$ & 嵒 & 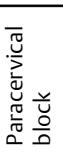 & 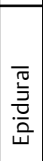 & 嵒 & 嵒 & 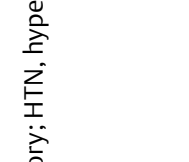 \\
\hline 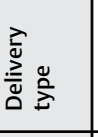 & 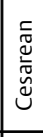 & is & 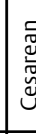 & 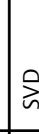 & 忩 & 党 & 公 & 空 & 忩 & 忿 & 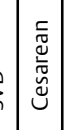 & 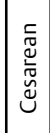 & 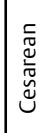 & is & 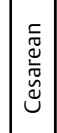 & 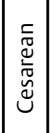 & 公 & 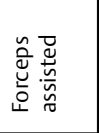 & 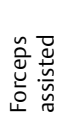 & 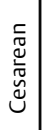 & 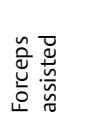 & 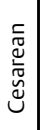 & 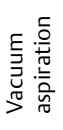 & 公 & 分 & $\stackrel{2}{\sim}$ & 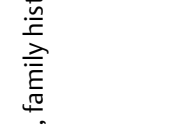 \\
\hline 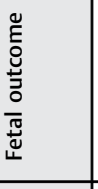 & $\stackrel{E}{E}$ & 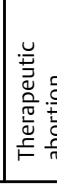 & 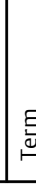 & E & 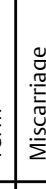 & 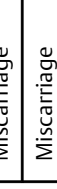 & 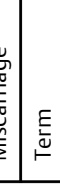 & $\stackrel{E}{\stackrel{E}{\omega}}$ & E & 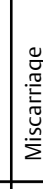 & 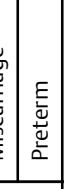 & 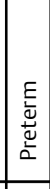 & $\stackrel{E}{\stackrel{E}{\omega}}$ & 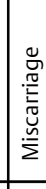 & \begin{tabular}{|l|}
$\stackrel{E}{\Xi}$ \\
$\stackrel{E}{E}$ \\
\end{tabular} & \begin{tabular}{|l|}
$E$ \\
$\stackrel{E}{W}$ \\
\end{tabular} & 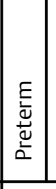 & 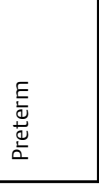 & 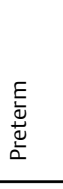 & $\underset{\stackrel{E}{O}}{\stackrel{E}{\circ}}$ & $\stackrel{E}{\stackrel{E}{\omega}}$ & $\frac{E}{U}$ & 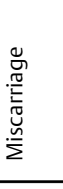 & $\stackrel{E}{E}$ & $\stackrel{E}{\stackrel{E}{\sigma}}$ & 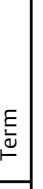 & $\begin{array}{l}\frac{\mathscr{c}}{\mathrm{o}} \\
\frac{0}{0}\end{array}$ \\
\hline 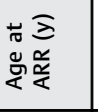 & $\stackrel{\infty}{\sim}$ & & & $\bar{m}$ & $\bar{m}$ & & & $\stackrel{\Delta}{\sim}$ & & $\approx$ & & 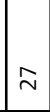 & $\approx$ & $\approx$ & & & $\approx$ & & & $\stackrel{\llcorner}{\sim}$ & N & $\stackrel{\sim}{\sim}$ & m & & $\bar{m}$ & & 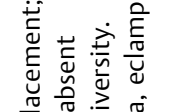 \\
\hline 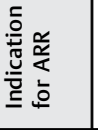 & $\varangle$ & & & $\varangle$ & 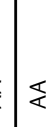 & & & $\varangle$ & & $\varangle$ & & $\varangle$ & $\varangle$ & \& & & & $\varangle$ & & & $\varangle$ & \& & 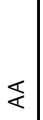 & $\varangle$ & & ₹ & & 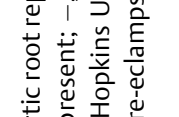 \\
\hline 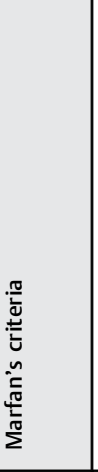 & 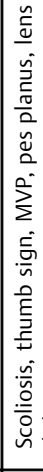 & 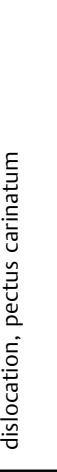 & & 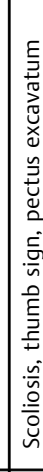 & 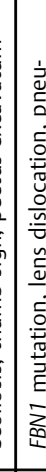 & 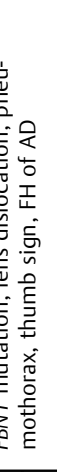 & & 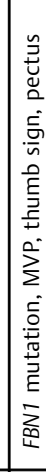 & 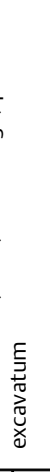 & & 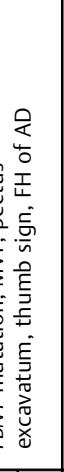 & 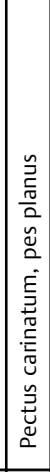 & 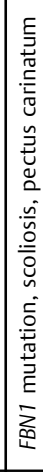 & 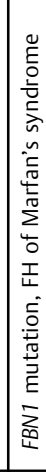 & & & 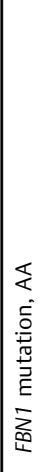 & & & 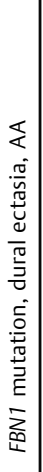 & 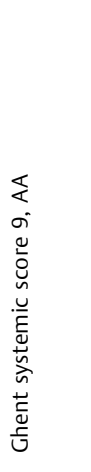 & 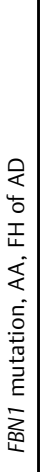 & 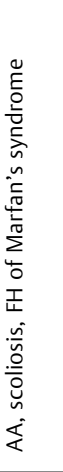 & & 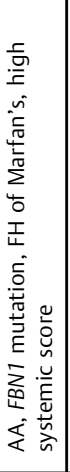 & & 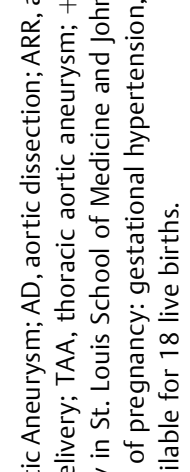 \\
\hline 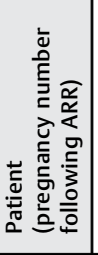 & $\underset{\ulcorner}{\Xi}$ & $\underset{-}{\widehat{\Xi}}$ & $\overline{\underline{m}}$ & ב & 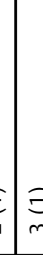 & $\begin{array}{l}n= \\
n\end{array}$ & $\frac{\widehat{m}}{m}$ & $\underset{\sigma}{\Xi}$ & $\frac{\widehat{a}}{\sigma}$ & E & {$\left[\begin{array}{l}a \\
\vdots \\
i n\end{array}\right.$} & $\mid \begin{array}{l}E \\
0 \\
0\end{array}$ & E & $E_{\infty}$ & $\begin{array}{c}\widehat{a} \\
\infty \\
\infty\end{array}$ & \begin{tabular}{|c|}
$\widehat{0}$ \\
$\infty$ \\
\end{tabular} & \begin{tabular}{|l|} 
\\
$\bar{\sigma}$ \\
\end{tabular} & $\begin{array}{l}\bar{a} \\
\sigma\end{array}$ & $\begin{array}{l}\widehat{\tilde{n}} \\
\sigma\end{array}$ & $\begin{array}{l}\bar{\Xi} \\
0 \\
\circ\end{array}$ & $\begin{array}{l}\Xi \\
\Xi\end{array}$ & $\begin{array}{l}\bar{N} \\
\simeq\end{array}$ & $\begin{array}{l}\text { E) } \\
\text { m } \\
m\end{array}$ & & 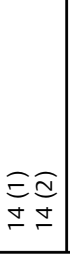 & & 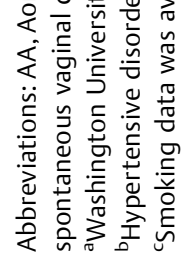 \\
\hline
\end{tabular}


Table 2 Characteristics of women with Marfan's syndrome in online survey and WU/JHH Marfan's clinics

\begin{tabular}{|c|c|}
\hline Variable & $\begin{array}{l}n=14 \text { Marfan's women, } \\
20 \text { live birth pregnancies }\end{array}$ \\
\hline $\begin{array}{l}\text { Age at aortic root } \\
\text { replacement }(y)\end{array}$ & $27.4 \pm 4.1$ \\
\hline $\begin{array}{l}\text { History of prior aortic } \\
\text { dissection }\end{array}$ & $2(14.3 \%)$ \\
\hline $\begin{array}{l}\text { Pregnancies following } \\
\text { aortic root replacement }\end{array}$ & $1.4 \pm 0.6$ \\
\hline Chronic hypertension & $3(21.4 \%)$ \\
\hline $\begin{array}{l}\beta \text {-blocker use per } \\
\text { pregnancy }\end{array}$ & $18(90 \%)$ \\
\hline Smoking per pregnancy ${ }^{c}$ & $3(16.7 \%)$ \\
\hline \multirow[t]{4}{*}{$\begin{array}{l}\text { Antithrombotic therapy } \\
\text { per pregnancy }\end{array}$} & $6(30 \%)$ \\
\hline & Aspirin (1) \\
\hline & Heparin (2) \\
\hline & $\begin{array}{l}\text { Warfarin (1) } \\
\text { Lovenox (2) }\end{array}$ \\
\hline $\begin{array}{l}\text { Hypertensive disorder } \\
\text { of pregnancy }{ }^{b}\end{array}$ & $1(5 \%)$ \\
\hline $\begin{array}{l}\text { Anesthesia use } \\
\text { during delivery }\end{array}$ & $\begin{array}{l}\text { Epidural } 18(90 \%) \\
\text { General } 1(5 \%) \\
\text { Spinal } 1(5 \%)\end{array}$ \\
\hline $\begin{array}{l}\text { Breastfeeding } \geq 2 \text { wk } \\
\text { per pregnancy }\end{array}$ & $14(70 \%)$ \\
\hline
\end{tabular}

Abbreviations: JHH, John Hopkins Hospital; WU, Washington University in St. Louis School of Medicine.

${ }^{a}$ Smoking data was available for 18 live births.

bHypertensive disorder of pregnancy includes gestational hypertension, preeclampsia, and eclampsia.

women reported their physician recommend against pregnancy following aortic root replacement.

\section{Literature Review}

A comprehensive literature review was performed identifying reports of pregnancy outcomes in women with Marfan's syndrome after prior aortic root replacement. We compiled data on 24 women with Marfan's syndrome who had 24 pregnancies after prior aortic root replacement ( - Table 3). ${ }^{4,7-17}$ Of these 24 pregnancies, there were 23 live births and one fetal demise due to aortic dissection in the second trimester. Three of the 24 women had undergone aortic root replacement for acute type A (ascending) aortic dissection, and 13 patients had elective aortic surgery for aneurysm. Indication for aortic root replacement was not known in eight women. Of the 22 women for whom further data are available, 8 (36.4\%) patients underwent a composite aortic valve and root replacement, 13 underwent valve-sparing root replacement (59.1\%), and 1 underwent aortic homograft root replacement. The use of $\beta$-blocker therapy was known in 11 women. Of those 11 women, 8 (72.7\%) used a $\beta$-blocker during pregnancy. Methods of delivery were reported in 16 pregnancies and included cesarean delivery in 13 women, spontaneous vaginal delivery in 2 , and forceps assisted in 1 .

Acute aortic dissection related to pregnancy was reported in 7 of 24 women with Marfan's syndrome (29.2\%) who had undergone prior aortic root replacement. ${ }^{4,7-10}$ Chavanon et al reported a case of a woman who suffered an acute type $B$ aortic dissection and worsening aortic regurgitation two days after delivery. ${ }^{9}$ McDermott et al reported one woman who sustained an acute type B dissection and severe hypertension in the second trimester, resulting in patient death and intrauterine fetal demise. ${ }^{7}$ Meijboom et al reported the case of a woman with a prior type A aortic dissection and composite aortic valve graft who suffered an acute type B aortic dissection at 27 weeks of gestation. ${ }^{8}$ She was treated with initial medical therapy, undergoing a cesarean delivery at 34 weeks, and subsequently elective aortic repair after delivery. Rossiter et al reported a patient with a prior acute type A dissection with chronic dissection of the descending thoracic aorta who suffered an extension of the chronic descending dissection 1 week postpartum. ${ }^{4}$ This patient used illicit drugs including cocaine during her pregnancy. Sayama reported five women with Marfan's syndrome who had pregnancy after aortic root replacement and strikingly, three of the five patients (60\%) in this series suffered a type B aortic dissection related to pregnancy. ${ }^{10}$

Regarding counseling from the literature review, some authors recommended against pregnancy if patients were deemed to have risk factors for aortic dissection, specifically a prior aortic dissection. ${ }^{4,14,15}$

\section{Discussion}

In our case series, there were no aortic dissections among 20 successful pregnancies in 14 women with Marfan's syndrome who had undergone aortic root replacement before pregnancy. Most of our patients did not have additional risk factors for a high-risk pregnancy (i.e., hypertension, smoking) which may have been protective against aortic dissection; all patients except one were prescribed and presumably compliant with $\beta$-blocker therapy. Patient 9 ( - Table 1) had a history of smoking and hypertension and developed a descending thoracic aneurysm after a second pregnancy. Whether hypertension, smoking, and multiparity were risk factors in her progressive aneurysm disease is unknown. The mode of delivery, the method of anesthesia, or breastfeeding was not related to aortic events.

Our report represents the largest series to date describing aortic outcomes related to pregnancy in women with Marfan's syndrome who had undergone prior aortic root replacement. Differing from high incidence from case reports in the literature, no women in our series suffered aortic dissection related to pregnancy.

There are several limitations to this study including the observational nature, small sample size, potential for sampling error, and recall bias. The patients surveyed and those from the Marfan syndrome clinics represent a survivor cohort, so the risk of aortic complications may be 
Table 3 Literature review of pregnancy outcomes in women with Marfan's syndrome following aortic root repair

\begin{tabular}{|c|c|c|c|c|c|c|c|c|}
\hline Reference & $n$ & $\begin{array}{l}\text { Indication } \\
\text { for ARR }\end{array}$ & ARR type & $\beta$-blocker & $\begin{array}{l}\text { Method of } \\
\text { delivery }\end{array}$ & Anesthesia & Aortic complications & Counseling \\
\hline Rossiter $^{4}$ & 1 & $\begin{array}{l}\text { Type A AD } \\
\text { extending into } \\
\text { thoracic aorta }\end{array}$ & CVG & Yes & Unknown & Unknown & $\begin{array}{l}\text { Extension of type } B A D \\
1 \text { wk postpartum }\end{array}$ & $\begin{array}{l}\text { Advised against } \\
\text { pregnancy; Patient used } \\
\text { illicit intravenous drugs, } \\
\text { including cocaine before } \\
\text { and during pregnancy }\end{array}$ \\
\hline Donnelly ${ }^{11}$ & 3 & $\overline{A A}$ & $\begin{array}{l}2 \text { VSRR } 1 \\
\text { CVG }\end{array}$ & Unknown & Unknown & Unknown & $\begin{array}{l}\text { No dissection but } \\
\text { worsened aortic } \\
\text { regurgitation in both } \\
\text { women with VSRR }\end{array}$ & Unknown \\
\hline McDermott $^{7}$ & 1 & $\mathrm{AA}$ & VSRR & Yes & NA & NA & $\begin{array}{l}\text { Fatal type B AD in } 2 \mathrm{nd} \\
\text { trimester; fetal demise, } \\
\text { SBP on presentation } \\
>200 \mathrm{~mm} \mathrm{Hg}\end{array}$ & $\begin{array}{l}\text { Preconception } \\
\text { counseling } \\
\text { recommended against } \\
\text { pregnancy before aortic } \\
\text { repair and reevaluation } \\
\text { after surgery }\end{array}$ \\
\hline Tutarel $^{12}$ & 1 & $\begin{array}{l}\text { Type A AD } \\
\text { extending into } \\
\text { iliac arteries }\end{array}$ & CVG & Yes & Cesarean & Unknown & $\begin{array}{l}\text { No further extension of } \\
\text { chronic dissection which } \\
\text { was confirmed by MRI in } \\
3^{\text {rd }} \text { trimester of } \\
\text { pregnancy }\end{array}$ & Unknown \\
\hline Williams $^{13}$ & 1 & $\mathrm{AA}$ & $\begin{array}{l}\text { Homo-graft } \\
\text { root }\end{array}$ & Yes & $\begin{array}{l}\text { Forceps- } \\
\text { assisted }\end{array}$ & Unknown & None & $\begin{array}{l}\text { Quoted risk of aortic root } \\
\text { dissection to be }<10 \%\end{array}$ \\
\hline Allyn ${ }^{14}$ & 2 & Unknown & Unknown & Unknown & Cesarean & $\begin{array}{l}\text { General } \\
\text { anesthesia }\end{array}$ & None & $\begin{array}{l}\text { Advised against } \\
\text { pregnancy if history } \\
\text { of AD or mechanical } \\
\text { aortic valve }\end{array}$ \\
\hline Omnes $^{15}$ & 3 & Unknown & 3 VSRR & Unknown & Cesarean & $\begin{array}{l}\text { General } \\
\text { anesthesia }\end{array}$ & None & $\begin{array}{l}\text { Advised against } \\
\text { pregnancy if history } \\
\text { of } A D \text { or mechanical } \\
\text { aortic valve }\end{array}$ \\
\hline Curry $^{16}$ & 3 & Unknown & $\begin{array}{l}1 \text { CVG } 2 \\
\text { VSRR }\end{array}$ & Unknown & Unknown & Unknown & None & Unknown \\
\hline Meijboom $^{8}$ & 2 & $\begin{array}{l}\text { 1-Type A AD } \\
\text { 2-AA }\end{array}$ & $2 C V G$ & Unknown & Cesarean & Unknown & $\begin{array}{l}\text { Patient with prior Type A } \\
\text { AD, developed a Type B } \\
\text { AD at } 27 \text { wk, underwent } \\
\text { surgical repair at } 34 \text { wk } \\
\text { delivery }\end{array}$ & $\begin{array}{l}28 \% \text { had not conceived } \\
\text { based on advice from } \\
\text { cardiologist }\end{array}$ \\
\hline Chavanon $^{9}$ & 1 & $\mathrm{AA}$ & VSRR & Yes & Cesarean & General & $\begin{array}{l}\text { Type B AD } 2 \mathrm{~d} \text { after } \\
\text { delivery and worsening } \\
\text { aortic regurgitation }\end{array}$ & Unknown \\
\hline Volach $^{17}$ & 1 & $\overline{A A}$ & CVG & Yes & Cesarean & Epidural & None & Unknown \\
\hline Sayama $^{10}$ & 5 & $\begin{array}{l}\text { 1-AA } \\
\text { 2-AA } \\
3-A A \\
4-A A \\
5-A A\end{array}$ & $\begin{array}{l}\text { 1-VSRR } \\
\text { 2-CVG } \\
\text { 3-VSRR } \\
\text { 4-VSRR } \\
\text { 5-VSRR }\end{array}$ & $\begin{array}{l}1 \text { 1-No } \\
\text { 2-No } \\
\text { 3-Yes } \\
4 \text {-Yes } \\
5 \text {-No }\end{array}$ & $\begin{array}{l}\text { 1-Cesarean } \\
\text { 2-Vaginal } \\
\text { 3-Cesarean } \\
\text { 4-Cesarean } \\
\text { 5-Vaginal }\end{array}$ & $\begin{array}{l}\text { 1-Unknown } \\
\text { 2-Epidural } \\
\text { 3-Unknown } \\
\text { 4-Unknown } \\
\text { 5-Epidural }\end{array}$ & $\begin{array}{l}\text { 1-None } \\
\text { 2-None } \\
\text { 3-Type B AD at } 36 \text { wk } \\
\text { 4-Type B AD } 5 \text { d } \\
\text { postpartum } \\
\text { 5-Type B AD 11 d } \\
\text { postpartum }\end{array}$ & Unknown \\
\hline
\end{tabular}

Abbreviations: AA, aortic aneurysm; AD, aortic dissection; ARR, aortic root replacement; CVG, composite valve graft; MRI, magnetic resonance imaging; N, number of pregnancies ARR; SBP, systolic blood pressure; VSRR, valve sparing root replacement.

underestimated. We were unable to assess for compliance with medications or other complications of pregnancy following valve sparing aortic root replacement, such as aortic regurgitation. Additionally, complete medical records were not available for all patients obtained from our survey and clinics at WU and JHH. Thus, the information gathered is subject to recall bias. Furthermore, two women (patients 2 and 6) in our study did not provide all data to satisfy the revised Ghent criteria for Marfan's syndrome ( - Table $\mathbf{1}){ }^{18}$ Thus, it is possible these subjects did not have Marfan's syndrome but a related aortopathy syndrome. Despite these limitations, our series is the largest to date examining aortic outcomes related to pregnancy in women with Marfan's syndrome with prior aortic root replacement.

Our results contrast with prior published reports. The results of the literature review may result in overestimation of the risk of aortic dissection related to pregnancy after aortic root replacement due to a reporting or publication bias. Importantly, one report had a very high percentage of pregnancy-related aortic dissections (60\%) which may create bias toward increased risk. ${ }^{10}$ The indication for aortic surgery was not reported in all women from the literature 
review limiting our ability to be certain about risks of aortic dissection related to prior aortic events. As compiled from our case series, the risk of aortic dissection related to pregnancy after root replacement in women with Marfan's syndrome is likely lower than that reported in the literature.

While many experts have recommended that women with Marfan's syndrome and aortic dilatation undergo elective aortic root replacement before contemplating pregnancy, the risk of acute aortic dissection during subsequent pregnancy is poorly understood. ${ }^{5}$ The National Registry of Genetically Triggered Thoracic Aortic Aneurysms and Cardiovascular Conditions (GenTAC) registry reported pregnancy-related aortic dissection risk (5.4 per 100 patient years) for women with Marfan's syndrome but did not include any women with pregnancy following aortic root replacement. ${ }^{19}$ Pregnancy in Marfan's syndrome is known to be a risk factor for aortic complications due to the underlying aortopathy and pregnancy-related molecular, hormonal, and hemodynamic changes. ${ }^{3,20}$ Most aortic dissections during pregnancy in these women occur in the third trimester and early postpartum period. ${ }^{3,11,19}$ This may be related to pregnancy-related hemodynamic or hormonal changes which may persist for several weeks postpartum. ${ }^{21}$ Alterations in elastic fibers and mucopolysaccharides have also been described during pregnancy. ${ }^{22}$ Aortic root growth rate in Marfan's syndrome is reported to accelerate during pregnancy but following pregnancy, the aortic root growth reverts to a prepregnancy rate. ${ }^{11}$ In patients with Marfan's syndrome who have undergone prior valve sparing root replacement, an increase in the degree aortic regurgitation during pregnancy and postpartum is reported. ${ }^{9,11}$

Prior aortic root replacement may be a risk factor for distal aortic events in Marfan's syndrome ${ }^{23}$ with late type B dissection being reported in approximately $9 \%$ of patients. ${ }^{24,25}$ Independent risk factors for dissection include prior root replacement, aortic dilatation, and decreased aortic distensibility while angiotensin receptor blockers were protective. ${ }^{26}$ It is hypothesized that abnormal elastic properties related to the aortic root vascular prosthesis may result in higher pulsatile forces on the native aortic arch and proximal descending aorta which can affect distal aortic events. ${ }^{24,26}$

Beta adrenergic blockers and angiotensin II receptor blockers are beneficial in managing aortic disease in Marfan's syndrome and $\beta$-blockers are recommended for women with Marfan's syndrome during pregnancy. ${ }^{5,6,27}$ Whether $\beta$ blockers have a protective benefit during pregnancy has not been studied. Angiotensin II receptor blockers are contraindicated during pregnancy due to their teratogenicity. Following aortic root replacement surgery, long-term $\beta$ blocker therapy is recommended in Marfan's syndrome to lessen aortic wall stress. ${ }^{5}$

Loeys-Dietz syndrome, sometimes initially diagnosed as Marfan's syndrome, has a more aggressive vascular phenotype than Marfan's syndrome and aortic dissection is reported related to pregnancy in this condition. ${ }^{23,28}$ Pregnancy following root replacement in Loeys-Dietz syndrome is reported and associated with a very high rate of distal aortic dissection. ${ }^{29}$

\section{Conclusion}

A multidisciplinary approach including cardiology, medical genetics, maternal fetal medicine, anesthesia (and cardiac surgery as appropriate) is recommended in the care of women with Marfan's syndrome contemplating pregnancy. Management should include $\beta$-blockers during pregnancy, control of hypertension and tobacco cessation. There are insufficient data available to inform counseling on breastfeeding. Imaging of the aorta should be performed prior to pregnancy, during pregnancy, and in the postpartum period. Women who have undergone valve-sparing root replacement should have echocardiography to evaluate for aortic regurgitation. Women and their physicians should be vigilant for signs and symptoms of acute aortic dissection during pregnancy and postpartum.

Women with Marfan's syndrome who have undergone prior aortic root replacement and desire pregnancy should be counseled that they remain at risk for distal aortic dissection related to pregnancy. The exact risk of pregnancy following elective root replacement is difficult to calculate but it is not zero. The risk is likely higher when aortic surgery was performed for an aortic dissection or if residual dissection is present than when performed for asymptomatic aneurysm disease. Discussion of the potential for aortic complications should take place prior to pregnancy for women with Marfan's syndrome who have undergone prior aortic surgery.

\section{Conflict of Interest}

None.

\section{Acknowledgments}

Funding for Dr. Dietz-this work was supported by funding from the Marfan Foundation, the Smilow Foundation, the Howard Hughes Medical Institute, and the National Institutes of Health (R01-AR41135). No other authors had funding support for this work.

\section{References}

1 Finkbohner R, Johnston D, Crawford ES, Coselli J, Milewicz DM. Marfan syndrome. Long-term survival and complications after aortic aneurysm repair. Circulation 1995;91(03):728-733

2 Lipscomb KJ, Smith JC, Clarke B, Donnai P, Harris R. Outcome of pregnancy in women with Marfan's syndrome. Br J Obstet Gynaecol 1997;104(02):201-206

3 Pacini L, Digne F, Boumendil A, et al. Maternal complication of pregnancy in Marfan syndrome. Int J Cardiol 2009;136(02):156-161

4 Rossiter JP, Repke JT, Morales AJ, Murphy EA, Pyeritz RE. A prospective longitudinal evaluation of pregnancy in the Marfan syndrome. Am J Obstet Gynecol 1995;173(05):1599-1606

5 Hiratzka LF, Bakris GL, Beckman JA, et al; American College of Cardiology Foundation/American Heart Association Task Force on Practice Guidelines; American Association for Thoracic Surgery; American College of Radiology; American Stroke Association; Society of Cardiovascular Anesthesiologists; Society for Cardiovascular Angiography and Interventions; Society of Interventional Radiology; Society of Thoracic Surgeons; Society for Vascular Medicine. 2010 ACCF/AHA/AATS/ACR/ASA/SCA/SCAI/SIR/STS/SVM Guidelines for the diagnosis and management of patients with 
thoracic aortic disease. A report of the American College of Cardiology Foundation/American Heart Association Task Force on Practice Guidelines, American Association for Thoracic Surgery, American College of Radiology, American Stroke Association, Society of Cardiovascular Anesthesiologists, Society for Cardiovascular Angiography and Interventions, Society of Interventional Radiology, Society of Thoracic Surgeons,and Society for Vascular Medicine. J Am Coll Cardiol 2010;55(14):e27-e129

6 Regitz-Zagrosek V, Blomstrom Lundqvist C, Borghi C, et al; European Society of Gynecology (ESG); Association for European Paediatric Cardiology (AEPC); German Society for Gender Medicine (DGesGM); ESC Committee for Practice Guidelines. ESC Guidelines on the management of cardiovascular diseases during pregnancy: the task force on the Management of cardiovascular diseases during Pregnancy of the European Society of Cardiology (ESC). Eur Heart J 2011;32(24):3147-3197

7 McDermott CD, Sermer M, Siu SC, David TE, Colman JM. Aortic dissection complicating pregnancy following prophylactic aortic root replacement in a woman with Marfan syndrome. Int J Cardiol 2007;120(03):427-430

8 Meijboom LJ, Drenthen W, Pieper PG, et al; ZAHARA investigators Obstetric complications in Marfan syndrome. Int J Cardiol 2006; 110(01):53-59

9 Chavanon O, Rama A, Leprince P, et al. Valve-sparing operation in a young woman with Marfan syndrome: a word of caution. J Thorac Cardiovasc Surg 2006;132(03):683-684

10 Sayama S, Takeda N, Iriyama T, et al. Peripartum type B aortic dissection in patients with Marfan syndrome who underwent aortic root replacement: a case series study. BJOG 2018;125(04): 487-493

11 Donnelly RT, Pinto NM, Kocolas I, Yetman AT. The immediate and long-term impact of pregnancy on aortic growth rate and mortality in women with Marfan syndrome. J Am Coll Cardiol 2012;60 (03):224-229

12 Tutarel O, Lotz J, Roentgen P, Drexler H, Meyer GP, Westhoff-Bleck M. Pregnancy in a Marfan patient with pre-existing aortic dissection. Int J Cardiol 2007;114(02):E36-E37

13 Williams A, Child A, Rowntree J, Johnson P, Donnai P. Marfan's syndrome: successful pregnancy after aortic root and arch replacement. BJOG 2002;109(10):1187-1188

14 Allyn J, Guglielminotti J, Omnes S, et al. Marfan's syndrome during pregnancy: anesthetic management of delivery in 16 consecutive patients. Anesth Analg 2013;116(02):392-398

15 Omnes S, Jondeau G, Detaint D, et al. Pregnancy outcomes among women with Marfan syndrome. Int J Gynaecol Obstet 2013;122 (03):219-223

16 Curry RA, Gelson E, Swan L, et al. Marfan syndrome and pregnancy: maternal and neonatal outcomes. BJOG 2014;121(05):610-617
17 Volach V, Elami A, Gilon D, Pollak A, Ginosar Y, Ezra Y. Pregnancy in Marfan syndrome after aortic root replacement: a case report and review of the literature. Congenit Heart Dis 2006;1(04):184-188

18 Loeys BL, Dietz HC, Braverman AC, et al. The revised Ghent nosology for the Marfan syndrome. J Med Genet 2010;47(07):476-485

19 Roman MJ, Pugh NL, Hendershot TP, et al; GenTAC InvestigatorsDietzHarry C.HabashiJenniferPrakashSiddharth K.MaslenCheryl L.SongHoward K.BavariaJoseph E.MilewskiKariannaWeinsaftJonathan W. McDonnellNazliAschFederico M.TolunayH. EserDesvigne-NickensPatriceTsengHungKronerBarbara L. Aortic complications associated with pregnancy in Marfan syndrome: the NHLBI National Registry of Genetically Triggered Thoracic Aortic Aneurysms and Cardiovascular Conditions (GenTAC). J Am Heart Assoc 2016;5(08):e004052

20 Pyeritz RE. Maternal and fetal complications of pregnancy in the Marfan syndrome. Am J Med 1981;71(05):784-790

21 Abbas AE, Lester SJ, Connolly H. Pregnancy and the cardiovascular system. Int J Cardiol 2005;98(02):179-189

22 Goland S, Barakat M, Khatri N, Elkayam U. Pregnancy in Marfan syndrome: maternal and fetal risk and recommendations for patient assessment and management. Cardiol Rev 2009;17(06): 253-262

23 Loeys BL, Schwarze U, Holm T, et al. Aneurysm syndromes caused by mutations in the TGF-beta receptor. N Engl J Med 2006;355 (08):788-798

24 den Hartog AW, Franken R, Zwinderman AH, et al. The risk for type B aortic dissection in Marfan syndrome. J Am Coll Cardiol 2015;65 (03):246-254

25 Song HK, Kindem M, Bavaria JE, et al; Genetically Triggered Thoracic Aortic Aneurysms and Cardiovascular Conditions Consortium. Long-term implications of emergency versus elective proximal aortic surgery in patients with Marfan syndrome in the Genetically Triggered Thoracic Aortic Aneurysms and Cardiovascular Conditions Consortium Registry. J Thorac Cardiovasc Surg 2012;143(02):282-286

26 Groenink M, den Hartog AW, Franken R, et al. Losartan reduces aortic dilatation rate in adults with Marfan syndrome: a randomized controlled trial. Eur Heart J 2013;34(45):3491-3500

27 Lacro RV, Dietz HC, Sleeper LA, et al; Pediatric Heart Network Investigators. Atenolol versus losartan in children and young adults with Marfan's syndrome. N Engl J Med 2014;371(22):2061-2071

28 Jondeau G, Ropers J, Regalado E, et al; Montalcino Aortic Consortium. International registry of patients carrying TGFBR1 or TGFBR2 mutations: results of the MAC (Montalcino Aortic Consortium). Circ Cardiovasc Genet 2016;9(06):548-558

29 Braverman AC, Moon MR, Geraghty P, Willing M, Bach C, Kouchoukos NT. Pregnancy after aortic root replacement in Loeys-Dietz syndrome: high risk of aortic dissection. Am J Med Genet A 2016; 170(08):2177-2180 